\title{
Qualidade da Consulta de Medicina Geral e Familiar Percecionada pelo Utente e pelo Médico
}

Ricardo Amaral*, Luiz Miguel Santiago**

\section{Resumo}

Introdução: A qualidade dos Serviços de Saúde e da consulta médica tem vindo a ser analisada em múltiplos trabalhos de investigação, tendo conduzido a alterações nos modelos de saúde. A satisfação do utente é um reflexo da qualidade dos Serviços de Saúde, devendo ser monitorizada e consequente na alteração dos problemas identificados. A consulta de Medicina Geral e Familiar (MGF) é das formas mais acessíveis dos cuidados de saúde, tendo como princípios base a horizontalidade, equidade e universalidade, sendo por isso particularmente importante na avaliação da qualidade dos cuidados de saúde.

Objetivos: Este trabalho teve como objetivo conhecer o nível da satisfação global dos utentes com a consulta e se os médicos são capazes de avaliar corretamente a satisfação dos utentes com a mesma.

Métodos: Foi utilizado um questionário validado de 11 itens, aplicado no final das consultas de Medicina Geral e Familiar a médicos e utentes.

Resultados: Numa amostra de conveniência de $n=144$, com uma taxa de resposta de $88,8 \%(n=128)$, maioritariamente do género feminino $(59,4 \%)$, ativa profissionalmente (56,3\%), e com formação superior a 9 anos de escolaridade para $50,8 \%$, os resultados demonstraram que os utentes estão satisfeitos com as consultas de MGF e que os médicos subavaliam a satisfação dos utentes sendo a comparação entre respostas de utentes e médicos de pior resultado para os médicos com elevado significado estatístico. De salientar os utentes considerarem o equipamento do consultório como o aspeto mais fraco.

\begin{abstract}
Background: The Health Service and medical consultation quality have both been analyzed in several investigation papers, which has resulted in adjustments to previous health models. Thus, patient satisfaction is proof of the quality of diferent Health Services, which should be monitored and thorough in the correction of the identified problems. The General Practice (GP) consultation is one of the most acessible forms of health care, and it has as core principles horizontality, equity and universality, therefore being particularly important in the evaluation of health care quality.
\end{abstract}

Goals: This paper had as main goals the appreciation of the global satisfaction of the patients with the consultation and also whether doctors are able to evaluate patient satisfaction accordingly.

Methods: It was used a validated questionnaire of 11 items, which was applied at the end of the General Practice consultation to both doctors and patients.

Results: In a convenience sample of $n=144$, with a response rate of $88,8 \%(n=128)$, the majority of which were of the female gender $(59,4 \%)$, professionaly active (56,3\%), and with education higher than 9 years $(50,8 \%)$, the results have demontrated that patients are generaly satisfied with GP consultations and that doctors undervalue patient satisfaction; also, the comparison of answers of patients and doctors has revealed a worst result for doctors with high statistical significance. It should be pointed that patients consider the medical office equipment as the weakest point of the consultation.

\footnotetext{
* Primeiro autor: Ricardo Amaral, Interno do Ano Comum no Centro Hospitalar Tondela-Viseu.

** Co-autor: Luiz Miguel Santiago, Especialista em MGF, USF Topázio PhD, Faculdade de Ciências da Saúde da Universidade da Beira Interior.
} 
Discussão: Outros estudos devem ser feitos para confirmar os resultados e identificar possíveis causas para a subavaliação da satisfação dos utentes por parte dos médicos e suas consequências.

Palavras-chave: Satisfação do utente, Qualidade dos cuidados de saúde, Consulta, Medicina geral e familiar.

\section{Introdução}

A qualidade dos Serviços de Saúde em geral e da consulta médica em particular apresenta-se como temática recorrente nas últimas décadas. Esta qualidade é entendida como uma necessidade intrínseca aos próprios serviços,,$^{1,2}$ tendo conduzido a alterações nas políticas e modelos de saúde ${ }^{2,3}$ e motivado múltiplos trabalhos de investigação. ${ }^{4,5}$ Assiste-se a uma crescente preocupação com a satisfação do utente, ${ }^{1,2}$ uma vez que este está cada vez mais informado e exigente, ${ }^{2}$ é a peça basilar do sistema e cada vez mais corresponsável pelo custo da saúde. Em Portugal, segundo indicadores da OCDE (Organização para a Cooperação e Desenvolvimento Económico), 29\% dos custos totais com saúde em 2011 foram financiados diretamente pelos utilizadores. ${ }^{6}$

A Medicina Geral e Familiar (MGF) enquanto o contacto mais próximo do utente com o Sistema de Saúde, apresentando nos seus atributos a universalidade e um acesso horizontal e sendo responsável pela medicina preventiva e promotora da saúde, ${ }^{2,5,7}$ além de prestação de cuidados curativos e de reabilitação, deve ser tida particularmente em conta na avaliação da qualidade dos serviços prestados. É também fundamental a sua melhoria contínua, visto esta refletir e contribuir para as condições económicas e sociais. ${ }^{8}$ Para isso, entre outras medidas anteriores, desde a criação do Serviço Nacional de Saúde, foi implementada em 2006 uma reforma nos cuidados de saúde primários em Portugal, com a legislação e posterior fundação de unidades de saúde com autonomia organizativa, funcional e técnica, com contratualização de carteiras de serviços e remuneração de acordo com o cumprimento de objetivos em determinados indicadores também contratualizados - Unidades de Saúde Familiar (USF) ${ }^{1}$, 2, 7, 9, 10; bem como a criação das Unidades de Cuidados de Saúde Personalizados (UCSP), sem as condições acima referidas, sujeitas apenas a cumprimento de indicadores, à data presente.

A consulta e, em particular, o binómio utente-médico constituem-se como o elemento mais importante a avaliar neste âmbito. Vários estudos mostram que a
Discussion: More studies must be carried out to confirm these results and identify possible causes for the aforementioned doctors undervaluation of patient satisfaction and its consequences.

Keywords: Patient satisfaction, Quality of health care, Consultation, General practice

consulta médica é o momento chave da prestação de cuidados de saúde, sendo o que mais afeta a satisfação do doente. . $^{31,12}$

Na maioria dos estudos apenas se avalia a satisfação do utente, ficando por saber se o médico é capaz de a prever corretamente.

Assim, neste trabalho, propomos estudar, com recurso a questionários validados, a apreciação da qualidade da consulta de Medicina Geral e Familiar do ponto de vista do utente e do médico, analisando e comparando a avaliação de cada um dos intervenientes.

\section{Métodos}

Após parecer positivo da Comissão de Ética da Administração Regional de Saúde (ARS) Centro e do Agrupamento de Centros de Saúde (ACeS) Baixo Mondego foram iniciados os contactos com os coordenadores das unidades de saúde - USF Topázio, USF Cruz de Celas, UCSP de Celas, USF Marquês de Marialva e UCSP de Cantanhede -, tendo-se o investigador deslocado às unidades na data combinada a realizar o estudo. Foram entregues no final da consulta, aos utentes maiores de 15 anos com capacidade de ler e escrever e que tenham aceitado participar, um consentimento informado com a descrição do estudo e o questionário validado com um quadro de caracterização de cada indivíduo da população por género, idade, atividade profissional, formação académica e tempo de espera pela consulta (Anexo 2 e 3) e 11 afirmações para as quais foi solicitada resposta de acordo com o grau de concordância com cada afirmação. Ao mesmo tempo, o médico que efetuou a consulta preencheu um questionário versando os mesmos pontos mas sob a sua perspetiva. Os questionários de médico e utente encontravam-se codificados para posterior emparelhamento. 0 investigador recolheu os questionários preenchidos pelos utentes e devidamente colocados em envelopes fechados, quando impossivel a entrega em mão, e os dos médicos, no final de todas as consultas. 
De forma a facilitar a análise foram criadas novas variáveis com os dados colhidos no quadro de caracterização do utente. Categorizou-se a idade em três grupos - de 16 a 34 anos, de 35 a 64 anos e mais de 65 anos -; a formação académica em dois grupos até ao $9^{\circ}$ ano inclusive, mais que o $9^{\circ}$ ano -; e a atividade profissional em dois grupos - ativo (rural, comércio, indústria, serviços, doméstica) e inativo (desempregado, reformado, estudante).

Foi elaborada uma base de dados para análise estatística descritiva e inferencial usando o software SPSS 15. Os testes estatísticos usados foram $U$ de Mann-Whitney - para avaliar as diferenças nas respostas de acordo com género (masculino/feminino), atividade (ativo/inativo) e formação (até $9^{\circ}$ ano/mais que $9^{\circ}$ ano); Kruskall-Wallis - para avaliar as diferenças nas respostas de acordo com grupo etário (até 34 anos, entre 35 e 64 anos e 65 ou mais anos) e tempo de espera pela consulta (até 10 minutos, 10 a 20 minutos, mais de 20 minutos). Para análise de diferenças na resposta às afirmações do questionário entre utentes e médicos foi utilizado o teste de sinais de Wilcoxon para amostras pareadas.

\section{Resultados}

Dos 144 questionários entregues foram recebidos 128 $(88,8 \%)-66 \%$ em USF e 34\% em UCSP. Com cerca de $59,4 \%$ de respondentes do género feminino, com idade média de 50,4 anos $\pm 16,77 ; 56,3 \%$ ativos profissionalmente e 50,8\% com formação superior ao $9^{\circ}$ ano.

O tempo de espera pela consulta foi inferior a 10 minutos para $39,3 \%$, entre 10 e 20 minutos para $27,6 \%$ e superior a 20 minutos para 33,1\%.

Na Tabela 1 encontram-se descritas a concordância com cada uma das afirmações do questionário por parte dos utentes. A concordância foi menor na questão 1. "O consultório está bem equipado". Houve apenas uma resposta de concordância nula na pergunta 4 ("Fizeram-me um exame por causa das minhas queixas"), pergunta onde foi também registada a maior taxa de não respostas. Globalmente os utentes concordaram totalmente ou muito com as afirmações indicando uma elevada satisfação com a consulta.

Tabela 1: Concordância com as afirmações do questionário por parte dos utentes.

\begin{tabular}{|c|c|c|c|c|}
\hline \multicolumn{5}{|c|}{ Concordância com as Afirmações - Utentes } \\
\hline \multirow[b]{2}{*}{ Afirmação } & \multicolumn{4}{|c|}{ Concordância (\%) } \\
\hline & Total & Muita & Pouca & Nenhuma \\
\hline 1. O consultório está bem equipado. $(n=125)$ & 45,6 & 49,6 & 4,8 & 0,0 \\
\hline $\begin{array}{l}\text { 2. O médico mostrou ter tempo para me ouvir sobre os meus } \\
\text { problemas. }(n=127)\end{array}$ & 82,7 & 16,5 & 0,8 & 0,0 \\
\hline 3. Pude falar sobre o que me trouxe à consulta. $(n=126)$ & 84,9 & 13,5 & 1,6 & 0,0 \\
\hline 4. Fizeram-me um exame por causa das minhas queixas. $(n=111)$ & 76,6 & 22,5 & 0,0 & 0,9 \\
\hline 5. O médico explicou-me os meus problemas. $(n=124)$ & 80,6 & 16,9 & 2,4 & 0,0 \\
\hline 6. Disseram-me a importância do que fazer para melhorar. $(n=125)$ & 80,0 & 19,2 & 0,8 & 0,0 \\
\hline 7. Julgo que o médico entendeu os meus problemas. $(n=126)$ & 84,1 & 15,1 & 0,8 & 0,0 \\
\hline 8. Percebi as informações que o médico me deu. $(n=126)$ & 82,5 & 16,7 & 0,8 & 0,0 \\
\hline 9. O ambiente para a consulta é agradável. $(n=128)$ & 75,0 & 25,0 & 0,0 & 0,0 \\
\hline 10. A consulta durou o tempo necessário. $(n=128)$ & 79,7 & 20,3 & 0,0 & 0,0 \\
\hline 11. Gostei da consulta. $(n=128)$ & 84,4 & 14,8 & 0,8 & 0,0 \\
\hline
\end{tabular}

Não se encontraram diferenças estatisticamente significativas na avaliação da consulta por parte dos utentes de acordo com as variáveis sociodemográficas (género, idade, atividade, formação ou tempo de espera) - Tabela 2. 
Tabela 2: Significância estatística - valor de $p$ - na avaliação de diferenças nas respostas nas concordâncias dos utentes segundo as variáveis sociodemográficas género, formação, atividade, idade e tempo de espera.

\begin{tabular}{|c|c|c|c|c|c|}
\hline & \multicolumn{5}{|c|}{ Variáveis Sociodemográficas } \\
\hline & Género $^{1}$ & Formação ${ }^{1}$ & Atividade $^{1}$ & Idade $^{2}$ & Tempo de Espera ${ }^{2}$ \\
\hline 1. O consultório está bem equipado. & 0,261 & 0,091 & 0,578 & 0,497 & 0,998 \\
\hline $\begin{array}{l}\text { 2. O médico mostrou ter tempo para me ouvir sobre } \\
\text { os meus problemas. }\end{array}$ & 0,18 & 0,614 & 0,881 & 0,440 & 0,452 \\
\hline 3. Pude falar sobre o que me trouxe à consulta. & 0,089 & 0,209 & 0,808 & 0,339 & 0,761 \\
\hline $\begin{array}{l}\text { 4. Fizeram-me um exame por causa das minhas } \\
\text { queixas. }\end{array}$ & 0,384 & 0,387 & 0,213 & 0,445 & 0,175 \\
\hline 5. O médico explicou-me os meus problemas. & 0,048 & 0,480 & 0,477 & 0,392 & 0,091 \\
\hline $\begin{array}{l}\text { 6. Disseram-me a importância do que fazer para } \\
\text { melhorar. }\end{array}$ & 0,203 & 0,537 & 0,991 & 0,640 & 0,136 \\
\hline 7. Julgo que o médico entendeu os meus problemas. & 0,150 & 0,376 & 0,325 & 0,470 & 0,473 \\
\hline 8. Percebi as informações que o médico me deu. & 0,357 & 0,064 & 0,563 & 0,887 & 0,753 \\
\hline 9 O ambiente para a consulta é agradável. & 0,026 & 0,876 & 0,581 & 0,778 & 0,295 \\
\hline 10. A consulta durou o tempo necessário. & 0,212 & 0,790 & 0,662 & 0,630 & 0,269 \\
\hline 11. Gostei da consulta. & 0,155 & 0,149 & 0,667 & 0,305 & 0,188 \\
\hline
\end{tabular}

${ }^{1} \mathrm{U}$ de Mann-Whitney; ${ }^{2}$ Kruskall-Wallis

A distribuição das respostas dos médicos às afirmações encontra-se descrita na Tabela 3. A menor concordância prevista pelos médicos foi na Afirmação 1.
Tabela 3: Concordância com as afirmações do questionário por parte dos médicos.

\begin{tabular}{|c|c|c|c|c|}
\hline \multicolumn{5}{|c|}{ Concordância com as Afirmações - Médicos } \\
\hline \multirow{2}{*}{$\begin{array}{l}\text { Afirmação } \\
\text { Julgo que o utente: }\end{array}$} & \multicolumn{4}{|c|}{ Concordância (\%) } \\
\hline & Total & Muita & Pouca & Nenhuma \\
\hline 1. Pensa que o consultório está bem equipado. $(n=139)$ & 7,9 & 85,6 & 6,5 & 0,0 \\
\hline $\begin{array}{l}\text { 2. Percebeu que demostrei ter tempo para o ouvir falar sobre os seus } \\
\text { problemas. }(n=142)\end{array}$ & 21,1 & 78,2 & 0,7 & 0,0 \\
\hline 3. Pensa que pôde falar sobre o que preocupava. $(n=140)$ & 14,3 & 82,8 & 2,9 & 0,0 \\
\hline 4. Percebeu que o examinei por causa dos seus problemas. $(n=137)$ & 15,3 & 83,2 & 1,5 & 0,0 \\
\hline 5. Percebeu que Ihe expliquei os seus problemas. $(n=139)$ & 18,0 & 80,6 & 1,4 & 0,0 \\
\hline $\begin{array}{l}\text { 6. Percebeu que Ihe expliquei a importância do que fazer para } \\
\text { melhorar. }(n=139)\end{array}$ & 15,1 & 82,7 & 2,2 & 0,0 \\
\hline 7. Ficou convencido de que entendi os seus problemas. $(n=140)$ & 15,7 & 81,4 & 2,9 & 0,0 \\
\hline 8. Percebeu as informações que Ihe dei. $(n=142)$ & 16,2 & 81,7 & 2,1 & 0,0 \\
\hline 9. Pensa que o ambiente para a consulta é agradável. $(n=142)$ & 9,2 & 88,0 & 2,8 & 0,0 \\
\hline 10. Pensa que a consulta durou o tempo necessário. $(n=140)$ & 10,7 & 86,4 & 2,9 & 0,0 \\
\hline 11. Gostou da consulta. $(n=142)$ & 10,6 & 86,6 & 2,8 & 0,0 \\
\hline
\end{tabular}


Obtiveram-se valores negativos de correlação entre a avaliação do doente e a prevista pelo médico com significância estatística para todos os pares de afirmações através do teste de Wilcoxon (Tabela 4), ou seja, os médicos previram as respostas dos seus utentes em valor mais baixo.
Tabela 4: Correlação entre as respostas às afirmações por utentes e médicos.

\begin{tabular}{|c|c|c|}
\hline \multicolumn{3}{|c|}{ Correlação entre as Respostas de Utentes e Médicos } \\
\hline Afirmação & $\mathbf{z}^{1}$ & $\mathrm{Sig}^{2}$ \\
\hline 1. O consultório está bem equipado. & $-5,73693$ & $<0,001$ \\
\hline 2. O médico mostrou ter tempo para me ouvir sobre os meus problemas. & $-8,52802$ & $<0,001$ \\
\hline 3. Pude falar sobre o que me trouxe à consulta. & $-9,03469$ & $<0,001$ \\
\hline 4. Fizeram-me um exame por causa das minhas queixas. & $-7,33629$ & $<0,001$ \\
\hline 5. O médico explicou-me os meus problemas. & $-7,77565$ & $<0,001$ \\
\hline 6. Disseram-me a importância do que fazer para melhorar. & $-8,39362$ & $<0,001$ \\
\hline 7. Julgo que o médico entendeu os meus problemas. & $-9,03061$ & $<0,001$ \\
\hline 8. Percebi as informações que o médico me deu. & $-8,90824$ & $<0,001$ \\
\hline 9. O ambiente para a consulta é agradável. & $-8,93322$ & $<0,001$ \\
\hline 10. A consulta durou o tempo necessário. & $-9,28061$ & $<0,001$ \\
\hline 11. Gostei da consulta. & $-9,41186$ & $<0,001$ \\
\hline
\end{tabular}

${ }^{1}$ Wilcoxon, ${ }^{2}$ Significância estatística - valor de $p$

\section{Discussão}

A amostra usada neste estudo pode conter vários vieses de amostragem por ser uma amostra de conveniência sujeita ao voluntarismo de médicos e utentes e de confundimento como acquiescence - concordar acriticamente - e social desirability - vontade de cumprir as normas sociais ou sair-se bem no estudo. ${ }^{14}$

Como pontos fortes do estudo temos a destacar o uso de um questionário validado e um investigador externo às unidades de saúde.

A elevada concordância demonstrada pelos utentes com as afirmações do questionário pode dever-se em parte aos vieses; no entanto, outros estudos já demonstraram elevada satisfação dos utentes com os cuidados de saúde primários em Portugal.2, 3, 4, 15, 16

A menor concordância dos utentes com a afirmação 1 ("O consultório está bem equipado") pode dever-se a desconhecimento das necessidades do consultório. Esse resultado já foi obtido noutros estudos. ${ }^{4}$ É ainda de referir que esta foi também a afirmação que menos concordância teve entre médicos o que sugere que os médicos reconhecem que as falhas de equipamentos nos consultórios são notórias para os utentes. Este resultado e hipóteses avançadas devem ser alvo de futuros estudos nomeadamente verificando in loco o equipamento dos consultórios.

Noutro estudo foi relatado um desfasamento entre as expectativas do doente acerca da realização do exame objetivo e o exame realizado pelo médico, o que pode ser justificativo juntamente com a inadequação no contexto da consulta para a menor taxa de respostas na afirmação 4 ("Fizeram-me um exame por causa das minhas queixas") quer nos questionários a utentes quer a médicos. ${ }^{16}$ Sugere-se em investigações vindouras utilizando este questionário a inclusão de um espaço de resposta livre em que o utente possa comentar o questionário e justificar as não respostas.

Ao contrário do sugerido por Santos ${ }^{17}$ e comprovado por Anderson ${ }^{12}$ não se mostrou que o tempo de espera influenciasse a avaliação global da consulta, resultado 
já obtido em outros estudos. ${ }^{13,15} \mathrm{Em}$ Arroyo $^{13}$ foi encontrada correlação entre um menor tempo de consulta e menor qualidade relatada pelo doente, algo que não foi possível avaliar no presente estudo por todos os questionários recebidos apresentarem concordância Total ou Muita com a afirmação "A consulta durou o tempo necessário", sendo necessária mais evidência para suportar essa hipótese.

Também ao contrário de outros estudos, variáveis demográficas como a escolaridade/grau de formação ou idade não pareceram influenciar a avaliação da consulta. ${ }^{2,5}$

O método de aferição da qualidade da consulta utilizado, por exigir respostas pré-definidas, não é capaz de averiguar outros pontos da consulta que possam ser problemáticos ou sobre os quais os utentes tenham uma opinião diferente. Para isso os grupos de discussão ou outro tipo de abordagem mais aberta podem ser mais úteis, como em "Os Centros de Saúde em Portugal - A Satisfação dos Utentes e dos Profissionais”. ${ }^{1}$

Os médicos não conseguem prever corretamente a avaliação da consulta feita pelos utentes, algo já teorizado. ${ }^{11} \mathrm{O}$ facto de os médicos preverem em baixa a avaliação que o utente faz da consulta pode ser justificado de várias formas, todas elas carecendo de confirmação com estudos posteriores por exemplo pedindo aos médicos para avançarem as suas explicações. Possíveis hipóteses são a humildade dos médicos, a constante pressão pela obtenção de melhores resultados, a motivação para fazer melhor e a possibilidade de o nível de objetivos para a consulta ser mais elevado para os médicos que para os utentes. Todas estas hipóteses singularmente ou combinadas podem resultar numa acérrima autocrítica.

Ainda como outra linha de investigação surge o desenvolvimento de um estudo que permita avaliar as implicações para utentes e médicos do reconhecimento em menor grau por parte dos médicos da satisfação sentida pelos utentes. Isto é, avaliar se devido a esta sub-avaliação dos médicos surgem resultados/consequências (output/outcome) em saúde diferentes para os utentes ou diferenças na satisfação profissional, ansiedade, burnout ou absentismo para os médicos.

\section{Conclusão}

A temática da qualidade da consulta e da satisfação do utente é recorrente. Neste trabalho propunha-se explorar a satisfação global dos utentes com a consulta, e se os médicos são capazes de avaliar corretamente essa satisfação. Trata-se de uma abordagem pouco utilizada e cuja relevância é desconhecida, carecendo de investigações complementares.

Este estudo permitiu assim concluir que os utentes estão satisfeitos com a consulta, avaliando-a de forma positiva; contudo, os médicos não conseguem prever o que os seus utentes sentem em relação à consulta, subestimando a avaliação dos mesmos sobre esta.

As variáveis género, idade, atividade e formação não influenciam a satisfação do utente em relação à consulta. O tempo de espera pela mesma também não exerce qualquer influência nessa avaliação.

Determinantes (como a humildade dos médicos ou nível de objetivos para a consulta superior para médicos que para utentes) e resultantes (resultados/consequências em saúde ou ansiedade, burnout ou absentismo dos médicos) da subavaliação da satisfação dos utentes por parte dos médicos constituem possíveis linhas de investigação futuras.

\section{Agradecimentos}

Agradeço ao meu coorientador Professor Doutor Luiz Miguel Santiago pela prontidão no esclarecimento de dúvidas e pelas sugestões dadas para este trabalho, e ao meu orientador, Professor Doutor Hernâni Caniço, pela sua disponibilidade. À Dr. ${ }^{a}$ Fátima Branco devo também um agradecimento pelo suporte inicial neste trabalho. À Dr. ${ }^{a}$ Inês Rosendo pelos comentários finais.

À minha família por todo o apoio ao longo do meu percurso académico, em especial aos meus avós Fernanda e José por todos os humildes conselhos e ensinamentos.

À Tânia Costa por fazer de mim alguém capaz de atingir o mais desejável objetivo. 


\section{Referências Bibliográficas}

1. Santos O, Biscaia A, Antunes AR, Craveiro I, Júnior A, Caldeira $R$, et al. Os Centros de Saúde em Portugal: A satisfação dos utentes e dos profissionais. 2007.

2. Machado CS de AM. Satisfação de Utentes do ACES Tâmega I - Baixo Tâmega. Instituto Politécnico do Porto; 2012.

3. Nascimento DMP. Satisfação Apercebida dos Utentes em Saúde. Universidade de Trás-os-Montes e Alto Douro; 2011.

4. Pimentel HJFM. Avaliação Da Satisfação Dos Utentes Em Relação Aos Centros De Saúde Do Serviço Regional De Saúde Dos Açores. Universidade dos Açores; 2010.

5. Guimarães MDCV. Satisfação do Utente e Qualidade Apercebida Avaliação no Centro de Saúde de Aldoar. Universidade Fernando Pessoa; 2009.

6. Oecd. Health at a glance 2013: OECD Indicators (OECD web site). 2013. Available at: http://dx.doi.org/10.1787/ health_glance-2013-en. Accessed February 20;2015

7. Afonso PB. Contratualização em ambiente de Cuidados de Saúde Primários. Rev Port Saúde Pública. 2010;9(desde 2003):59-64.

8. FendallNR.DeclarationofAlma-Ata.Lancet.1978;2(September 1978).

9. Melo M, Sousa JC De. Os Indicadores de Desempenho Contratualizados com as USF: Um ponto da situação no actual momento da Reforma. Rev Port Clínica Geral. 2011;28-34.

10. Rocha P, Sá A. Reforma da Saúde Familiar em Portugal: avaliação da implantação; Family Health Reform in Portugal: analysis of its implementation. Ciência e saúde coletiva [Internet]. 2011;2853-63. Available at: http://bases.bireme. br/cgi-bin/wxislind.exe/iah/online/?IsisScript=iah/iah.xis\&s rc $=$ google\&base $=$ LILACS\&lang $=p \&$ nextAction $=$ Ink\&exprSear $c h=591239$ \&indexSearch=ID. Accessed February 20;2015

\section{Conflito de Interesses}

Os autores declaram não ter conflitos de interesses.

\section{Endereço para Correspondência:}

Ricardo Amaral

Email: ricaja@gmail.com
11. Urdan AT. A qualidade de serviços médicos na perspectiva do cliente. RAE Rev Adm Empres. 2001;41(4):44-55.

12. Anderson RT, Camacho FT, Balkrishnan R. Willing to wait?: the influence of patient wait time on satisfaction with primary care. BMC Health Serv Res. 2007;7.

13. Arroyo CS. Qualidade de Serviços de Assistência à Saúde: o tempo de atendimento da consulta médica. Universidade de São Paulo, Faculdade de Economia, Administração e Contabilidade; 2007.

14. Furnham A. Response bias, social desirability and dissimulation. Personal indiviual Differ. 1986;7.

15. Almeida JMM. Descrição dos tempos de espera e sua relação com o grau de satisfação nos utentes da Unidade de Saúde de Aldoar. Universidade do Porto, Instituto de Ciências Biomédicas Abel Salazar; 2009.

16. Pinto D, Coutinho SS, Rezende C. Cumprimento de expectativas e satisfação com a consulta de Medicina Geral e Familiar. Rev Port Clin Geral. 2009;25:405-17.

17. Santos T, Rosendo I, Pimenta G, Martins D, Francisco MP, Neto M da G, et al. Qualidade da consulta de Medicina Geral e Familiar: conseguem os médicos julgar corretamente a qualidade. Rev Bras Med Família e Comunidade. 2007;3(9):13-20. 


\section{ANEXOS}

\section{ANEXO 1. Consentimento Informado}

Eu, Ricardo José Cardoso do Amaral, aluno nº 2009016926 do Mestrado Integrado em Medicina da Faculdade de Medicina da Universidade de Coimbra, encontro-me a realizar um estudo sobre a qualidade da consulta de Medicina Geral e Familiar percebida pelo utente e pelo médico.

A consulta e o contacto médico-doente estão intimamente ligados à qualidade dos cuidados de saúde. Logo para meIhorar os cuidados de saúde a opinião sobre a consulta da perspetiva tanto do doente como do médico é fundamental.

Peço-Ihe que responda a este Questionário, o coloque no envelope fechado e o deposite na caixa indicada.

Garantimos-Ihe que ninguém saberá as suas respostas. A análise global será depois publicitada, sendo ainda possível a sua divulgação científica.

Mesmo que tenha aceite preencher as 11 perguntas do questionário pode, a qualquer momento deixar de o responder.

Com os nossos agradecimentos,

0 investigador, 


\section{ANEXO 2. Questionário Utente}

Preencha s.f.f. colocando uma cruz no espaço correspondente ao termo que the parecer mais adequado, sobre se concorda com a afirmação escrita.

\begin{tabular}{|c|c|c|}
\hline Idade: & Idade __ anos & \\
\hline Género: & $\square$ Masculino & $\square$ Feminino \\
\hline Formação (a mais elevada que detém): & $\begin{array}{l}\square \text { Sabe ler e escrever } \\
\square 9^{\circ} \text { ano ( } 6^{\text {a }} \text { classe) } \\
\left.\square 12^{\circ} \text { ano ( } 7^{\circ} \text { ano }\right)\end{array}$ & $\begin{array}{l}\square \text { Técnica } \\
\square \text { Superior }\end{array}$ \\
\hline $\begin{array}{l}\text { Actividade em que ocupa a quase } \\
\text { totalidade do seu tempo e / ou em que } \\
\text { ganha quase todo o dinheiro mensal: }\end{array}$ & $\begin{array}{l}\square \text { Rural } \\
\square \text { Comércio } \\
\square \text { Indústria } \\
\square \text { Serviços }\end{array}$ & $\begin{array}{l}\square \text { Doméstica } \\
\square \text { Desempregado } \\
\square \text { Reformado } \\
\square \text { Estudante }\end{array}$ \\
\hline $\begin{array}{l}\text { Tempo e espera pela consulta desde } \\
\text { que se inscreveu: }\end{array}$ & $\begin{array}{l}\square \text { Até } 10 \text { minutos } \\
\square \text { Mais que } 20 \text { minutos }\end{array}$ & $\square$ Entre 10 e 20 minutos \\
\hline
\end{tabular}

\begin{tabular}{|c|c|c|c|c|c|}
\hline \multirow[t]{2}{*}{ Afirmação } & \multicolumn{5}{|c|}{ Concordância } \\
\hline & Total & Muita & Pouca & Nenhuma & $\begin{array}{l}\text { Não se } \\
\text { Aplica/NR }\end{array}$ \\
\hline 1. O consultório está bem equipado. & $\square$ & $\square$ & $\square$ & $\square$ & $\square$ \\
\hline $\begin{array}{l}\text { 2. O médico mostrou ter tempo para me ouvir } \\
\text { sobre os meus problemas. }\end{array}$ & $\square$ & $\square$ & $\square$ & $\square$ & $\square$ \\
\hline $\begin{array}{l}\text { 3. Pude falar sobre o que me trouxe à } \\
\text { consulta. }\end{array}$ & $\square$ & $\square$ & $\square$ & $\square$ & $\square$ \\
\hline $\begin{array}{l}\text { 4. Fizeram-me um exame por causa das } \\
\text { minhas queixas. }\end{array}$ & $\square$ & $\square$ & $\square$ & $\square$ & $\square$ \\
\hline 5. O médico explicou-me os meus problemas. & $\square$ & $\square$ & $\square$ & $\square$ & $\square$ \\
\hline $\begin{array}{l}\text { 6. Disseram-me a importância do que fazer } \\
\text { para melhorar. }\end{array}$ & $\square$ & $\square$ & $\square$ & $\square$ & $\square$ \\
\hline $\begin{array}{l}\text { 7. Julgo que o médico entendeu os meus } \\
\text { problemas. }\end{array}$ & $\square$ & $\square$ & $\square$ & $\square$ & $\square$ \\
\hline 8. Percebi as informações que o médico me deu. & $\square$ & $\square$ & $\square$ & $\square$ & $\square$ \\
\hline 9. O ambiente para a consulta é agradável. & $\square$ & $\square$ & $\square$ & $\square$ & $\square$ \\
\hline 10. A consulta durou o tempo necessário. & $\square$ & $\square$ & $\square$ & $\square$ & $\square$ \\
\hline 11. Gostei da consulta. & $\square$ & $\square$ & $\square$ & $\square$ & $\square$ \\
\hline
\end{tabular}


ANEXO 3. Questionário (Médico)

\begin{tabular}{|c|c|c|c|c|c|}
\hline Afirmação & & Con & cia & & \\
\hline $\begin{array}{l}\text { Julgo que o paciente / doente / utente / } \\
\text { consulente... }\end{array}$ & Total & Muita & Pouca & Nenhuma & $\begin{array}{c}\text { Não se } \\
\text { Aplica/NR }\end{array}$ \\
\hline 1. Pensa que o consultório está bem equipado. & $\square$ & $\square$ & $\square$ & $\square$ & $\square$ \\
\hline $\begin{array}{l}\text { 2. Percebeu que demostrei ter tempo para o } \\
\text { ouvir falar sobre os seus problemas. }\end{array}$ & $\square$ & $\square$ & $\square$ & $\square$ & $\square$ \\
\hline $\begin{array}{l}\text { 3. Pensa que pôde falar sobre o que } \\
\text { preocupava. }\end{array}$ & $\square$ & $\square$ & $\square$ & $\square$ & $\square$ \\
\hline $\begin{array}{l}\text { 4. Percebeu que o examinei por causa dos } \\
\text { seus problemas. }\end{array}$ & $\square$ & $\square$ & $\square$ & $\square$ & $\square$ \\
\hline $\begin{array}{l}\text { 5. Percebeu que Ihe expliquei os seus } \\
\text { problemas. }\end{array}$ & $\square$ & $\square$ & $\square$ & $\square$ & $\square$ \\
\hline $\begin{array}{l}\text { 6. Percebeu que the expliquei a importância do } \\
\text { que fazer para melhorar. }\end{array}$ & $\square$ & $\square$ & $\square$ & $\square$ & $\square$ \\
\hline $\begin{array}{l}\text { 7. Ficou convencido de que entendi os seus } \\
\text { problemas. }\end{array}$ & $\square$ & $\square$ & $\square$ & $\square$ & $\square$ \\
\hline 8. Percebeu as informações que Ihe dei. & $\square$ & $\square$ & $\square$ & $\square$ & $\square$ \\
\hline $\begin{array}{l}\text { 9. Pensa que o ambiente para a consulta é } \\
\text { agradável. }\end{array}$ & $\square$ & $\square$ & $\square$ & $\square$ & $\square$ \\
\hline $\begin{array}{l}\text { 10. Pensa que a consulta durou o tempo } \\
\text { necessário. }\end{array}$ & $\square$ & $\square$ & $\square$ & $\square$ & $\square$ \\
\hline 11. Gostou da consulta. & $\square$ & $\square$ & $\square$ & $\square$ & $\square$ \\
\hline
\end{tabular}

